PROCEEDINGS OF THE

AMERICAN MATHEMATICAL SOCIETY

Volume 133, Number 1, Pages 167-174

S 0002-9939(04)07516-1

Article electronically published on June 2, 2004

\title{
ON A GENERALIZED CORONA PROBLEM ON THE UNIT DISC
}

\author{
JORDI PAU
}

(Communicated by Juha M. Heinonen)

\begin{abstract}
Let $g, f_{1}, \ldots, f_{n} \in H^{\infty}$. We give a sufficient condition on the size of a function $g$ in order for it to be in the ideal generated by $f_{1}, \ldots, f_{n}$. In particular, this improves Cegrell's result on this problem.
\end{abstract}

\section{INTRODUCTION}

Let $\mathbb{D}$ be the unit disc in the complex plane, and let $H^{\infty}=H^{\infty}(\mathbb{D})$ be the Banach algebra of bounded analytic functions on $\mathbb{D}$. Carleson's corona theorem says that the unit disc is dense in the space $M_{H^{\infty}}$ of maximal ideals of $H^{\infty}$ with the weak-star topology. This result is equivalent to the following fact: if we have functions $f_{1}, \ldots, f_{n} \in H^{\infty}$ such that

$$
\sum_{j=1}^{n}\left|f_{j}(z)\right| \geq \delta>0, \quad \forall z \in \mathbb{D}
$$

then there exist solutions $g_{1}, \ldots, g_{n} \in H^{\infty}$ of the equation

$$
\sum_{j=1}^{n} f_{j} g_{j}=1
$$

In order to generalize this result, it is natural to ask if it is possible to replace the function 1 by an arbitrary function $g \in H^{\infty}$; that is, one asks if the condition

$$
|g(z)| \leq C \sum_{j=1}^{n}\left|f_{j}(z)\right|, \quad \forall z \in \mathbb{D},
$$

implies that the function $g$ belongs to the ideal $I$ generated by $f_{1}, \ldots, f_{n}$. Condition (11) is clearly a necessary condition, but an example given by Rao (see [Ra] shows that the answer is, in general, negative. Thus the following problem arises naturally.

Received by the editors January 31, 2003 and, in revised form, September 10, 2003.

2000 Mathematics Subject Classification. Primary 30D55; Secondary $46 J 15$.

Key words and phrases. $H^{p}$-spaces, corona problems, Carleson measure.

The author is supported by the EU Research Training Network HPRN-CT-2000-00116, and partially supported by SGR grant 2001SGR00431 and DGICYT grant PB98-0872.

(C)2004 American Mathematical Society 
Problem A. Let $h$ be a positive continuous function on $[0, \infty)$ increasing in a neighbourhood of zero, and let $g, f_{1}, \ldots, f_{n} \in H^{\infty}$. For which functions $h$ does the condition

$$
|g(z)| \leq h\left(\left|f_{1}(z)\right|+\cdots+\left|f_{n}(z)\right|\right), \quad \forall z \in \mathbb{D},
$$

imply that the function $g$ is in the ideal generated by $f_{1}, \ldots, f_{n}$ ?

For functions of the form $h(s)=s^{\alpha}$, with $\alpha \geq 1$, the problem is completely solved. For $1 \leq \alpha<2$, a variation of Rao's example shows that the answer is negative, and for $\alpha>2$, work of Wolff, Cegrell and others gives an affirmative answer (see [Ce1], Ga]). The problem for $\alpha=2$ was an old question of Wolff, which remained open for twenty years. However, Treil (see [Tr) has recently shown (using a connection with the best estimates of the solutions of the corona theorem) that the answer is, in general, negative.

In $[\mathrm{Li}$, Lin gave an affirmative answer for this problem for the function

$$
h(s)=s^{2}(-\log s)^{-(3 / 2+\varepsilon)}
$$

with $\varepsilon>0$, and in $\mathrm{Ce} 2$ Cegrell established the following strongest known positive case for this problem.

Theorem A (Cegrell). Let $f_{1}, \ldots, f_{n} \in H^{\infty}$ with $|f(z)|^{2}=\sum_{j=1}^{n}\left|f_{j}(z)\right|^{2}>0$, for all $z \in \mathbb{D}$. Then, Problem $A$ has an affirmative answer for

$$
h(s)=\frac{s^{2}}{(-\log s)^{3 / 2}(\log (-\log s))^{3 / 2} \log \log (-\log s)} .
$$

Our main result below is an improvement of Cegrell's theorem.

Theorem 1. Let $k:(0,1) \rightarrow[0, \infty)$ be a nondecreasing bounded continuous function such that $k(x) / x$ is nonincreasing and

$$
\int_{0}^{1} \frac{k(x)}{x}|\log x| d x<+\infty
$$

and let $H(x)=\sqrt{k(x) \int_{0}^{x} \frac{k(s)}{s} d s}$. Furthermore, let $g, f_{1}, \ldots, f_{n} \in H^{\infty}$, where $0<|f|^{2}:=\sum_{j=1}^{n}\left|f_{j}\right|^{2}<1$. Then the condition

$$
|g| \leq|f|^{2} H\left(|f|^{2}\right)
$$

implies the existence of solutions $g_{1}, \ldots, g_{n} \in H^{\infty}$ of the equation

$$
g=f_{1} g_{1}+\cdots+f_{n} g_{n} .
$$

For example, if we take $k(x)=|\log x|^{-2}(\log |\log x|)^{-3 / 2}$, we see that Problem A has an affirmative answer for the function

$$
h(s)=s^{2}(-\log s)^{-3 / 2}(\log (-\log s))^{-1},
$$

and this clearly improves Cegrell's result.

For $1 \leq p<\infty$, let $H^{p}$ be the Hardy space of analytic functions in the unit disc such that

$$
\|f\|_{p}^{p}=\sup _{0<r<1} \int_{0}^{2 \pi}\left|f\left(r e^{i \theta}\right)\right|^{p} \frac{d \theta}{2 \pi}<\infty .
$$


It is well known that an analytic function $f$ belongs to $H^{p}$ if and only if the nontangential maximal function $M f\left(e^{i \theta}\right)=\sup \{|f(z)|: z \in \Gamma(\theta)\}$ belongs to the usual Lebesgue space $L^{p}(\mathbb{T})$, where

$$
\Gamma(\theta):=\left\{z \in \mathbb{D}:\left|e^{i \theta}-z\right|<(1+\alpha)(1-|z|)\right\}
$$

is the Stolz angle with vertex at $e^{i \theta}$ and fixed aperture $\alpha>0$ (the choice of $\alpha$ is irrelevant here), and $\mathbb{T}$ denotes the unit circle. Several $H^{p}$ versions of the corona theorem have been considered. Concretely, one is interested in conditions on functions $f_{1}, \ldots, f_{n} \in H^{\infty}$ such that the equation

$$
1=f_{1} g_{1}+\cdots+f_{n} g_{n}
$$

has solutions $g_{1}, \ldots, g_{n}$ in $H^{p}$. If $|f|^{2}=\sum_{j=1}^{n}\left|f_{j}\right|^{2}$ and $|g|^{2}=\sum_{j=1}^{n}\left|g_{j}\right|^{2}$, it follows from (3) that $1 \leq|f||g|$, and hence $M\left(|f|^{-1}\right) \in L^{p}(\mathbb{T})$ is a necessary condition. We note that when $p=\infty$, this is the usual corona condition. However, for $1 \leq p<\infty$, this condition is far from being sufficient. In $[\mathrm{ABN}]$, it is shown that, for any $\varepsilon>0$, the stronger condition $M\left(|f|^{-2+\varepsilon}\right) \in L^{p}(\mathbb{T})$ is not sufficient. Our next result is the $H^{p}$ version of Theorem [1]

Theorem 2. Let $k$ be as in Theorem 11, let $H(x)=\left(k(x) \int_{0}^{x} k(s) / s d s\right)^{1 / 2}$ and let $1 \leq p<\infty$. Given functions $g, f_{1}, \ldots, f_{n} \in H^{\infty}$, the condition

$$
M\left(\frac{g}{|f|^{2} H\left(|f|^{2}\right)}\right) \in L^{p}(\mathbb{T})
$$

implies the existence of solutions $g_{1}, \ldots, g_{n} \in H^{p}$ of the equation

$$
g=f_{1} g_{1}+\cdots+f_{n} g_{n} .
$$

Finally, we want to remark that in both theorems, only the behavior of the function $k$, and hence $H$, near zero is essential.

\section{Carleson measures And the $\bar{\partial}$-EQuation}

Solutions of the $\bar{\partial}$-equation with boundary control will be of vital importance in the proofs of the main theorems, and Carleson measures play an important role in obtaining these solutions. We recall that a positive Borel measure $\mu$ on $\mathbb{D}$ is called a Carleson measure if there exists a constant $C$ such that

$$
\int_{\mathbb{D}}|h|^{2} d \mu \leq C\|h\|_{2}^{2}
$$

for every function $h$ in the Hardy space $H^{2}$. It is well known that Carleson measures are those positive measures $\mu$ for which there exists a constant $A$ such that

$$
\mu(Q) \leq A l(Q)
$$

for every Carleson square $Q$ defined by

$$
Q=\left\{r e^{i \theta} \in \mathbb{D}: 1-r<l(Q),\left|\theta-\theta_{0}\right|<l(Q)\right\} .
$$

Denote by $N(\mu)=\sup \{\mu(Q) / l(Q)\}$ the Carleson norm of $\mu$, where the supremum is taken over all Carleson squares $Q$. The operators $\partial$ and $\bar{\partial}$ are defined by

$$
\partial f=\frac{\partial f}{\partial z}=\frac{1}{2}\left(\frac{\partial f}{\partial x}-i \frac{\partial f}{\partial y}\right), \quad \bar{\partial} f=\frac{\partial f}{\partial \bar{z}}=\frac{1}{2}\left(\frac{\partial f}{\partial x}+i \frac{\partial f}{\partial y}\right) .
$$

By the Cauchy-Riemann equations, a function $f$ is analytic if and only if $\bar{\partial} f=0$. Recall that we can rewrite the Laplacian operator as $\Delta=4 \partial \bar{\partial}$. We need the 
following result of T. Wolff on the existence of bounded solutions of the $\bar{\partial}$-equation (see, for example, Ga] p. 322).

Lemma 1. Let $G(z)$ be bounded and $C^{1}$ on the disc $\mathbb{D}$, and assume that the measures $d \mu(z)=|G(z)|^{2} \log \frac{1}{|z|} d x d y$ and $d \sigma(z)=|\partial G(z)| \log \frac{1}{|z|} d x d y$ are Carleson measures. Then there exists a function $u \in C(\overline{\mathbb{D}}) \cap C^{1}(\mathbb{D})$ such that $\bar{\partial} u=G$ and

$$
\|u\|_{L^{\infty}(\mathbb{T})} \leq C_{1} N(\mu)^{1 / 2}+C_{2} N(\sigma) .
$$

We will also need an $L^{p}$-version of the Wolff criteria. The next lemma is a refinement of the version given in $[\mathrm{ABN}]$.

Lemma 2. Let $1 \leq p<\infty$, and let $G$ be a $C^{1}$ function in $\overline{\mathbb{D}}$ such that:

(a) $G=\varphi \psi$, where $M(\varphi) \in L^{p}(\mathbb{T})$, and $|\psi(z)|^{2} \log \frac{1}{|z|} d x d y$ is a Carleson measure;

(b) for every function $k \in H^{q}$, where $1 / p+1 / q=1$, we have

$$
\int_{\mathbb{D}}\left|k(z)\left\|\partial G(z) \mid \log \frac{1}{|z|} d x d y \leq B\right\| k \|_{q} .\right.
$$

Then there exists a function $u \in C(\overline{\mathbb{D}}) \cap C^{1}(\mathbb{D})$ such that $\bar{\partial} u=G$ and

$$
\int_{0}^{2 \pi}\left|u\left(e^{i \theta}\right)\right|^{p} d \theta \leq C
$$

where $C$ depends only on the $L^{p}$-norm of $M(\varphi)$, the constant $B$ and the Carleson norm of the measure of $(a)$.

Proof. Let $q$ be the conjugate exponent of $p, 1<q \leq \infty$. By duality,

$$
\inf \left\{\|b\|_{p}: \bar{\partial} b=G\right\}=\sup \left\{\left|\frac{1}{2 \pi} \int_{0}^{2 \pi} F k d \theta\right|: k \in H^{q}, k(0)=0,\|k\|_{q} \leq 1\right\}
$$

where $F$ is a priori solution, say the one given by the Cauchy kernel, which is continuous on $\overline{\mathbb{D}}$. By Green's formula,

$$
\begin{gathered}
\frac{1}{2 \pi} \int_{0}^{2 \pi} F k d \theta=\frac{1}{2 \pi} \int_{\mathbb{D}} \Delta(F k) \log \frac{1}{|z|} d x d y \\
=\frac{2}{\pi} \int_{\mathbb{D}} k^{\prime}(z) G(z) \log \frac{1}{|z|} d x d y+\frac{2}{\pi} \int_{\mathbb{D}} k(z) \partial G(z) \log \frac{1}{|z|} d x d y=I_{1}+I_{2} .
\end{gathered}
$$

It is proved in $\left[\mathrm{ABN}\right.$ that if $|\psi|^{2} \log \frac{1}{|z|}$ is a Carleson measure with Carleson norm $K$, then

$$
\int_{\mathbb{D}}\left|k^{\prime}(z)\right||\varphi(z)||\psi(z)| \log \frac{1}{|z|} d x d y \leq C\|k\|_{H^{q}}\|M \varphi\|_{p} K
$$

where $C$ is an absolute constant. This implies the required bound for $I_{1}$, and the boundness of $I_{2}$ follows from condition $(b)$.

The following lemma can be found in $\mathrm{Ni}$. For completeness we will give a proof here.

Lemma 3. Let $u \in C^{2}(\overline{\mathbb{D}})$ be a bounded subharmonic function. Then

$$
d \lambda(z)=\Delta u(z) \log \frac{1}{|z|} d x d y
$$

is a Carleson measure with Carleson norm bounded by $2 \pi e\|u\|_{\infty}$. 
Proof. By considering the function $b(z)=u(z)+\|u\|_{\infty}$ we can assume that our function $u$ is positive. Let $h \in H^{2}$. Then, for $t>0$,

$$
\begin{aligned}
\Delta\left(|h|^{2} e^{t u}\right) & =4\left|h^{\prime}\right|^{2} e^{t u}+|h|^{2} \Delta e^{t u}+8 \operatorname{Re}\left(\bar{\partial}\left(|h|^{2}\right) \partial e^{t u}\right) \\
& =4\left|h^{\prime}\right|^{2} e^{t u}+|h|^{2}\left(4 t^{2}|\partial u|^{2}+t \Delta u\right) e^{t u}+8 t e^{t u} \operatorname{Re}(h \bar{\partial} h \partial u) \\
& =t|h|^{2} e^{t u} \Delta u+e^{t u}|2 \partial h+2 t h \partial u|^{2} \\
& \geq t|h|^{2} e^{t u} \Delta u \geq t|h|^{2} \Delta u .
\end{aligned}
$$

Thus we have

$$
\int_{\mathbb{D}}|h(z)|^{2} \Delta u(z) \log \frac{1}{|z|} d x d y \leq \inf _{t>0} \frac{1}{t} \int_{\mathbb{D}} \Delta\left(|h(z)|^{2} e^{t u(z)}\right) \log \frac{1}{|z|} d x d y,
$$

which, by Green's formula, is bounded by

$$
\inf _{t>0} \frac{1}{t} \int_{\partial \mathbb{D}}|h|^{2} e^{t u} \leq \inf _{t>0} \frac{2 \pi}{t}\left\|e^{t u}\right\|_{\infty}\|h\|_{2}^{2}=2 \pi e\|u\|_{\infty}\|h\|_{2}^{2} .
$$

(The last identity is obtained by computation of the minimum of $t^{-1}\left\|e^{t u}\right\|_{\infty}$, which is attained at the point $t_{0}=1 /\|u\|_{\infty}$.) Hence the measure $\lambda$ is a Carleson measure with Carleson norm bounded by $2 \pi e\|u\|_{\infty}$.

Given functions $f_{1}, \ldots, f_{n} \in H^{\infty}$, we write $|f|^{2}=\sum_{i=1}^{n}\left|f_{i}\right|^{2}$, and $\left|f^{\prime}\right|^{2}=$ $\sum_{i=1}^{n}\left|f_{i}^{\prime}\right|^{2}$. The next result is the key for the proof of Theorems 1 and 2 .

Lemma 4. Let $k:(0,1) \rightarrow[0, \infty)$ be a bounded continuous function such that $\int_{0}^{1} \frac{k(x)}{x}|\log x| d x<\infty$. Let $f_{1}, \ldots, f_{n} \in H^{\infty}$ with $0<|f|^{2}<1$. Then the measures

(a) $\frac{\left|\partial\left(|f|^{2}\right)\right|^{2}}{|f|^{4}} k\left(|f|^{2}\right) \log \frac{1}{|z|} d x d y$,

(b) $\frac{|f|^{2}\left|f^{\prime}\right|^{2}-\left|\partial\left(|f|^{2}\right)\right|^{2}}{|f|^{4}}\left(\int_{0}^{|f|^{2}} \frac{k(s)}{s} d s\right) \log \frac{1}{|z|} d x d y$

are Carleson measures with Carleson norm bounded by $C \int_{0}^{1} \frac{k(s)}{s}|\log s| d s$.

We note that when $n=1$, part $(b)$ is vacuous and part $(a)$ is a known result that is also true for bounded analytic functions vanishing in $\mathbb{D}$ (see [ABN]).

Proof. Consider the function

$$
U(z)=\log |f(z)| \int_{0}^{|f(z)|^{2}} \frac{k(s)}{s} d s+\int_{0}^{|f(z)|^{2}} \frac{k(s)}{s}|\log s| d s .
$$

It clearly satisfies

$$
0 \leq U(z) \leq 2 \int_{0}^{|f(z)|^{2}} \frac{k(s)}{s}|\log s| d s
$$

and a computation gives

$$
\frac{1}{4} \Delta U(z)=S(z)
$$

where

$$
S=\frac{\left|\partial\left(|f|^{2}\right)\right|^{2}}{|f|^{4}} k\left(|f|^{2}\right)+\frac{|f|^{2}\left|f^{\prime}\right|^{2}-\left|\partial\left(|f|^{2}\right)\right|^{2}}{|f|^{4}}\left(\int_{0}^{|f|^{2}} \frac{k(s)}{s} d s\right) .
$$

Now, applying Lemma 3, we obtain the desired conclusion. 


\section{Proof of Theorem 1}

The proof follows standard arguments with the use of Lemma 4 as a new ingredient. By a standard normal families argument, we can assume that the functions $f_{1}, \ldots, f_{n}$ are analytic in a neighborhood of the closed unit disc. For $j=1, \ldots, n$ we define

$$
\varphi_{j}(z)=\frac{\overline{f_{j}(z)}}{|f(z)|^{2}} .
$$

We see that the functions $\varphi_{j}$ belong to $\mathcal{C}^{\infty}(\mathbb{D})$ and satisfy the equation $\sum_{j=1}^{n} f_{j} \varphi_{j}=$ 1. For $j, k=1, \ldots, n$, let

$$
G_{j k}=\varphi_{j} \bar{\partial} \varphi_{k}
$$

Assume that for $j, k=1, \ldots, n$ we can solve the $\bar{\partial}$-equations

$$
\bar{\partial} u_{j k}=g G_{j k}
$$

with $\left\|u_{j k}\right\|_{L^{\infty}(\mathbb{T})} \leq M$. Then, for $j=1, \ldots, n$, the functions

$$
g_{j}=g \varphi_{j}+\sum_{k=1}^{n}\left(u_{j k}-u_{k j}\right) f_{k}
$$

are bounded, satisfy $\bar{\partial} g_{j}=0$ and so are analytic, and satisfy the equation

$$
g=\sum_{j=1}^{n} f_{j} g_{j}
$$

It only remains to show that (5) has bounded solutions. To see this, we will use Lemma 1, Fix $j, k$, and denote $G_{j k}$ by $G$. A computation gives

$$
G_{j k}=\frac{\bar{f}_{j}}{|f|^{6}} \sum_{l \neq k} f_{l}\left(\overline{f_{l} f_{k}^{\prime}-f_{k} f_{l}^{\prime}}\right)
$$

and

$$
\sum_{j, k=1}^{n}\left|f_{k}^{\prime} f_{j}-f_{k} f_{j}^{\prime}\right|^{2}=|f|^{2}\left|f^{\prime}\right|^{2}-\left|\partial\left(|f|^{2}\right)\right|^{2}
$$

By (6) and (7),

$$
|G| \leq 2 \frac{\left(|f|^{2}\left|f^{\prime}\right|^{2}-\left|\partial\left(|f|^{2}\right)\right|^{2}\right)^{1 / 2}}{|f|^{4}}
$$

Using (8) and our condition on the size of $|g|$ we see that

$$
|g G|^{2} \leq \frac{\left(|f|^{2}\left|f^{\prime}\right|^{2}-\left|\partial\left(|f|^{2}\right)\right|^{2}\right)}{|f|^{4}}\left(\int_{0}^{|f|^{2}} \frac{k(s)}{s} d s\right) k\left(|f|^{2}\right),
$$

and because $k$ is bounded,

$$
|g(z) G(z)|^{2} \log \frac{1}{|z|} d x d y
$$

is a Carleson measure by Lemma 4,

We have $\partial(g G)=g^{\prime} G+g \partial G$, and since $|g| \leq|f|^{2} H\left(|f|^{2}\right)$, we have that the measure $|g(z) \partial G(z)| \log \frac{1}{|z|} d x d y$ is a Carleson measure by the following result.

Lemma 5. The measure $|f(z)|^{2} H\left(|f(z)|^{2}\right)|\partial G(z)| \log \frac{1}{|z|} d x d y$ is a Carleson measure. 
To prove this, we note that $k\left(|f|^{2}\right) \leq \int_{0}^{|f|^{2}} \frac{k(s)}{s} d s$, since the function $k(x) / x$ is nonincreasing. Also, a computation gives

$$
|\partial G| \leq 2|G| \frac{\left|\partial\left(|f|^{2}\right)\right|}{|f|^{2}}+\frac{|f|^{2}\left|f^{\prime}\right|^{2}-\left|\partial\left(|f|^{2}\right)\right|^{2}}{|f|^{6}}
$$

So, by (91) and then (8),

$$
\begin{aligned}
|f|^{2} H\left(|f|^{2}\right)|\partial G| & \leq 2 H\left(|f|^{2}\right)|G|\left|\partial\left(|f|^{2}\right)\right|+\frac{\left(|f|^{2}\left|f^{\prime}\right|^{2}-\left|\partial\left(|f|^{2}\right)\right|^{2}\right)}{|f|^{4}} \int_{0}^{|f|^{2}} \frac{k(s)}{s} d s \\
& \leq 2 \frac{\left|\partial\left(|f|^{2}\right)\right|^{2}}{|f|^{4}} k\left(|f|^{2}\right)+2 \frac{|f|^{2}\left|f^{\prime}\right|^{2}-\left|\partial\left(|f|^{2}\right)\right|^{2}}{|f|^{4}}\left(\int_{0}^{|f|^{2}} \frac{k(s)}{s} d s\right),
\end{aligned}
$$

and the result follows by Lemma 4 .

It remains to check that

$$
\left|g^{\prime}(z) G(z)\right| \log \frac{1}{|z|} d x d y
$$

is a Carleson measure. Let $h \in H^{2}$. Then

$$
\int_{\mathbb{D}}|h(z)|^{2}\left|\left(g^{\prime} G\right)(z)\right| \log \frac{1}{|z|} d x d y=\int_{A}+\int_{\mathbb{D} \backslash A}=I_{1}+I_{2},
$$

where $A=\left\{z:\left.|g(z) \leq| f(z)\right|^{5}\right\}$. For $z \in A$ we have

$$
\left|\left(g^{\prime} G\right)(z)\right| \leq \frac{\left|g^{\prime}(z)\right|^{2}}{|g(z)|}+\frac{\left|f^{\prime}(z)\right|^{2}}{|f(z)|} .
$$

Since for $F \in H^{\infty}$, the measure $\frac{\left|F^{\prime}(z)\right|^{2}}{|F(z)|} \log \frac{1}{|z|} d x d y$ is Carleson (see [Ga], p. 327, or apply Lemma 4 with $k(x)=x^{1 / 2}$ ), we see that $I_{1} \leq C_{1}\|h\|_{H^{2}}^{2}$, by (4). To estimate $I_{2}$, let

$$
B\left(|f|^{2}\right)=\frac{\left(|f|^{2}\left|f^{\prime}\right|^{2}-\left|\partial\left(|f|^{2}\right)\right|^{2}\right)}{|f|^{4}}\left(\int_{0}^{|f|^{2}} \frac{k(s)}{s} d s\right) .
$$

Since $k$ is nondecreasing, we see that

$$
\begin{aligned}
\left|g^{\prime} G\right| & \leq\left|g^{\prime}\right|^{2}|g|^{-2} k\left(|f|^{2}\right)+B\left(|f|^{2}\right) \\
& \leq\left|g^{\prime}\right|^{2}|g|^{-2} s\left(|g|^{2}\right)+B\left(|f|^{2}\right)
\end{aligned}
$$

in $\mathbb{D} \backslash A$, where $s(x)=k\left(x^{1 / 5}\right)$. One easily verifies that $s$ satisfies the condition $\int_{0}^{1} \frac{s(x)}{x}|\log x| d x<\infty$. Then $I_{2} \leq C_{2}\|h\|_{H^{2}}^{2}$ by Lemma 4 and (4). Hence the measure

$$
\left|\left(g^{\prime} G\right)(z)\right| \log \frac{1}{|z|} d x d y
$$

is a Carleson measure. By Lemma 1 the proof is complete.

\section{Proof of Theorem 2}

For $j, k=1, \ldots, n$, let $G_{j k}=\varphi_{j} \bar{\partial} \varphi_{k}$, where $\varphi_{j}=\bar{f}_{j}|f|^{-2}$. As in the proof of Theorem 1, it is sufficient to solve the $\bar{\partial}$-equations $\bar{\partial} u_{j k}=g G_{j k}$, with $\left\|u_{j k}\right\|_{L^{p}(\mathbb{T})} \leq$ $M$. For this, we will make use of Lemma 2, Fix $j, k$, and for ease of notation, denote $G_{j k}$ by $G$. We can write $g G$ in the form $g G=\phi \psi_{1}$, where $\phi=g|f|^{-2}\left(H\left(|f|^{2}\right)\right)^{-1}$ and $\psi_{1}=|f|^{2} H\left(|f|^{2}\right) G$. By hypothesis, $M(\phi) \in L^{p}(\mathbb{T})$, and the proof of Theorem 1] shows that

$$
\left|\psi_{1}(z)\right|^{2} \log \frac{1}{|z|} d x d y
$$


is a Carleson measure. So condition $(a)$ of Lemma 2 is satisfied. To check condition (b), let $k \in H^{q}$, where $1 / p+1 / q=1$. We have $\partial(g G)=g^{\prime} G+g \partial G$, and we can write $|g \partial G|$ as $|\phi| \psi_{2}$, where $\psi_{2}=|f|^{2} H\left(|f|^{2}\right)|\partial G|$. By Lemma [5] the measure

$$
d \mu(z)=\psi_{2}(z) \log \frac{1}{|z|} d x d y
$$

is a Carleson measure. Then

$$
\begin{aligned}
\int_{\mathbb{D}}|k(z)||(g \partial G)(z)| \log \frac{1}{|z|} d x d y & \leq\left(\int_{\mathbb{D}}|\phi|^{p} d \mu\right)^{1 / p}\left(\int_{\mathbb{D}}|k|^{q} d \mu\right)^{1 / q} \\
& \leq C\|M(\phi)\|_{L^{p}(\mathbb{T})}\|k\|_{H^{q}},
\end{aligned}
$$

since, if $\mu$ is a Carleson measure and $M \psi \in L^{p}(\mathbb{T})$, then $\int_{\mathbb{D}}|\psi|^{p} d \mu \leq\|M \psi\|_{L^{p}(\mathbb{T})}^{p}$ (see Ga, p. 32). An argument similar to that in the proof of Theorem 1 shows that

$$
\int_{\mathbb{D}}|k(z)|\left|\left(g^{\prime} G\right)(z)\right| \log \frac{1}{|z|} d x d y \leq C\|k\|_{H^{q}}
$$

and condition $(b)$ of Lemma 2 is satisfied. This completes the proof.

\section{REFERENCES}

[ABN] E. Amar, J. Bruna and A. Nicolau, On $H^{p}$-solutions of the Bezout equation, Pacific J. Math. 171: 2 (1995), 297-307. MR.97g:30036

[Ce1] U. Cegrell, A generalization of the corona theorem in the unit disc, Math. Z. 203 (1990), 255-261. MR91h:30059

[Ce2] U. Cegrell, Generalisations of the corona theorem in the unit disc, Proc. Roy. Irish Acad. 94 (1994), 25-30. MR 95k:30069

[Ga] J.B. Garnett, Bounded Analytic Functions, Academic Press, New York, 1981. MR $83 \mathrm{~g}: 30037$

[Li] K.C. Lin, On the constants in the corona theorem and ideals of $H^{\infty}$, Houston J. Math. 19 (1993), 97-106. MR94j:30033

[Ni] N.K. Nikol'skii, Treatise on the shift operator, Grund. der Math. Wissen., 273 (1986). MR $87 \mathrm{i}: 47042$

[Ra] K.V. Rao, On a generalized corona problem, J. Anal. Math. 18 (1967), 277-278. MR 35:1795

[Tr] S. Treil, Estimates in the corona theorem and ideals of $H^{\infty}:$ a problem of T. Wolff, J. Analyse Math. 87 (2002), 481-495. MR.2003k:30077

Departament de Matemàtiques, Universitat Autònoma de Barcelona, 08193 BellaTERRA, SPAIN

E-mail address: jpau@mat.uab.es 